\title{
Beam loss caused by edge focusing of injection bump magnets and its mitigation in the 3-GeV rapid cycling synchrotron of the Japan Proton Accelerator Research Complex
}

\author{
H. Hotchi, ${ }^{*}$ N. Tani, Y. Watanabe, H. Harada, S. Kato, K. Okabe, \\ P. K. Saha, F. Tamura, and M. Yoshimoto \\ Accelerator Division, J-PARC Center, Japan Atomic Energy Agency (JAEA), \\ Tokai, Naka, Ibaraki 319-1195, Japan \\ (Received 30 August 2015; published 13 January 2016)
}

\begin{abstract}
In the 3-GeV rapid cycling synchrotron of the Japan Proton Accelerator Research Complex, transverse injection painting is utilized not only to suppress space-charge induced beam loss in the low energy region but also to mitigate foil scattering beam loss during charge-exchange injection. The space-charge induced beam loss is well minimized by the combination of modest transverse painting and full longitudinal painting. But, for sufficiently mitigating the foil scattering part of beam loss, the transverse painting area has to be further expanded. However, such a wide-ranging transverse painting had not been realized until recently due to beta function beating caused by edge focusing of pulsed injection bump magnets during injection. This beta function beating additionally excites random betatron resonances through a distortion of the lattice superperiodicity, and its resultant deterioration of the betatron motion stability causes significant extra beam loss when expanding the transverse painting area. To solve this issue, we newly installed pulse-type quadrupole correctors to compensate the beta function beating. This paper presents recent experimental results on this correction scheme for suppressing the extra beam loss, while discussing the beam loss and its mitigation mechanisms with the corresponding numerical simulations.
\end{abstract}

DOI: 10.1103/PhysRevAccelBeams.19.010401

\section{INTRODUCTION}

The 3-GeV rapid cycling synchrotron (RCS) of the Japan Proton Accelerator Research Complex (J-PARC) is the world's highest class of high-power pulsed proton driver aiming for a 1-MW output beam power [1]. As shown in Fig. 1, a $400-\mathrm{MeV} \mathrm{H}^{-}$beam from the injector linac is delivered to the RCS injection point, where it is multiturn charge-exchange injected through a $340-\mu \mathrm{g} / \mathrm{cm}^{2}$-thick hybrid type boron-mixed carbon stripping foil [2] over a period of $0.5 \mathrm{~ms}$. RCS accelerates the injected protons up to $3 \mathrm{GeV}$ with a repetition rate of $25 \mathrm{~Hz}$, providing the $3-\mathrm{GeV}$ proton beam to the Material and Life Science Experimental Facility (MLF) and to the following 50-GeV Main Ring Synchrotron (MR) by switching the beam destination pulse by pulse. The main RCS parameters are summarized in Table. I.

RCS was beam commissioned in October 2007 [3] and made available for the user program in December 2008 with an output beam power of $4 \mathrm{~kW}$. Since then, the RCS beam power ramp-up has steadily proceeded as per progressions in beam tuning and hardware improvements

\footnotetext{
*hotchi.hideaki@jaea.go.jp

Published by the American Physical Society under the terms of the Creative Commons Attribution 3.0 License. Further distribution of this work must maintain attribution to the author(s) and the published article's title, journal citation, and DOI.
}

[4-8]. Recently injector linac upgrades were completed, by which the injection energy was upgraded from $181 \mathrm{MeV}$ to the design value of $400 \mathrm{MeV}$ in 2013, and then the injection peak current was increased from $30 \mathrm{~mA}$ to the design value of $50 \mathrm{~mA}$ in 2014. By these series of injector linac upgrades, RCS now has all the hardware parameters to realize its design performance. Thus RCS is in the final beam power ramp-up phase at the moment; the routine output beam power has been increased to $500 \mathrm{~kW}$ to date,

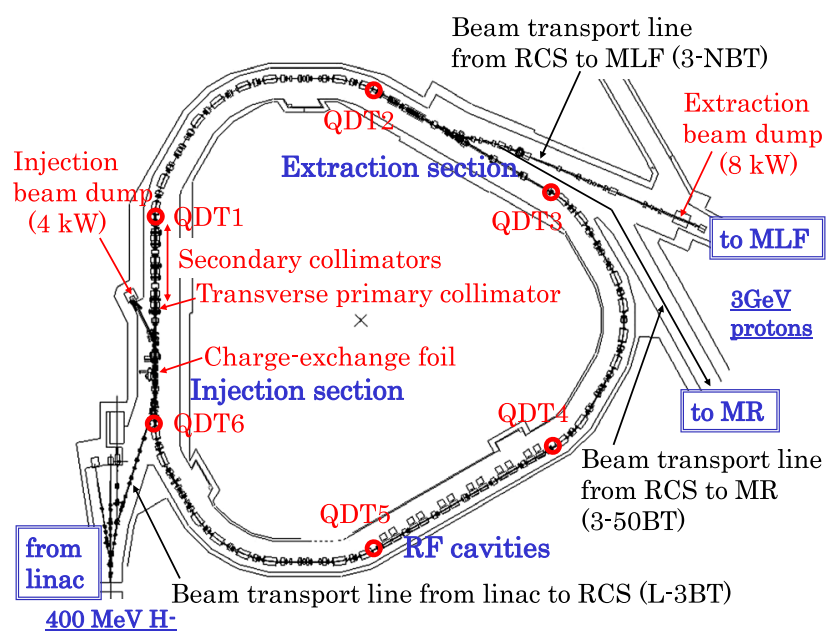

FIG. 1. Schematic view of RCS, where the red circles show the locations of quadrupole correctors (QDTs). 
TABLE I. RCS design parameters.

\begin{tabular}{lc}
\hline \hline Circumference & $348.333 \mathrm{~m}$ \\
Superperiodicity & 3 \\
Injected particles & $\mathrm{H}^{-}$ \\
Injection energy & $400 \mathrm{MeV}$ \\
Injection period & $0.5 \mathrm{~ms}$ \\
Injection turns & 307 \\
Injection peak current & $50 \mathrm{~mA}$ \\
Chopper beam-on duty factor of the injection & $53.3 \%$ \\
$\quad$ beam & \\
Unnormalized transverse emittance of the & $4 \pi \mathrm{mm} \mathrm{mrad}$ \\
$\quad$ injection beam & \\
Momentum spread of the injection beam & $\pm 0.1 \%$ \\
Extraction energy & $3 \mathrm{GeV}$ \\
Repetition rate & $25 \mathrm{~Hz}$ \\
Acceleration time & $20 \mathrm{~ms}$ \\
Ramping pattern & $\mathrm{Sinusoidal}$ \\
Transition energy & $9.2 \mathrm{GeV}$ \\
Harmonic number & 2 \\
Number of bunches & 2 \\
Number of particles per pulse & $8.33 \times 10^{13}$ \\
Output beam power & $1 \mathrm{MW}$ \\
Momentum acceptance & $\pm 1 \%$ \\
Ring acceptance & $486 \pi \mathrm{mm} \mathrm{mrad}$ \\
Collimator aperture & $324 \pi \mathrm{mm} \mathrm{mrad}$ \\
Collimator capability & $4 \mathrm{~kW}$ \\
Betatron tunes in this work & $(6.45,6.38)$ \\
\hline \hline
\end{tabular}

and from now on, it will be further ramped up toward the design value of $1 \mathrm{MW}$ step by step.

The most important issues in realizing such a MW-class high-power beam operation are controlling and minimizing beam loss to keep machine activations within a permissible level. The most critical beam loss in RCS arises from the space-charge effect in the low energy region, but now it is nearly minimized by employing transverse and longitudinal injection painting $[4,8]$. The beam loss that still remains is mainly from foil scattering during charge-exchange injection, which is inevitable as long as the charge-exchange foil is used. Most of the foil scattering beam losses are localized in the collimator section, and thus, no serious problem has been encountered to date. But some of them with large scattering angles are lost right downstream of the foil, leading to relatively high machine activations [9]. The residual radiation level detected on the chamber surface two hours after shutdown of the $400-\mathrm{kW}$ routine beam operation was around $15 \mathrm{mSv} / \mathrm{h}$. This value should be around $40 \mathrm{mSv} / \mathrm{h}$ if the output beam power is increased to $1 \mathrm{MW}$ as is. This radiation level is still considered to be within the acceptable level if assuming enough cooling time, but it has to be reduced as low as possible in terms of preserving a better hands-on-maintenance environment, because there are many devices in the injection section that often need maintenance.

The foil scattering beam loss can be mitigated by further expanding the transverse painting area, because transverse painting acts to reduce the foil hitting rate during injection, as is explained later. The transverse painting emittance was set to $100 \pi \mathrm{mm}$ mrad so far. Though this painting area is enough to minimize space-charge induced beam loss in combination with longitudinal painting [10,11], it has to be further expanded to $150 \pi \mathrm{mm}$ mrad or more to sufficiently mitigate the foil scattering beam loss. However, such a wide-ranging transverse painting had not been realized until recently due to beta function beating caused by edge focusing of pulsed injection bump magnets during injection. This beta function beating additionally excites random betatron resonances through a distortion of the lattice superperiodicity. This deterioration of the betatron motion stability causes significant extra beam loss when expanding the transverse painting area. To mitigate the foil scattering beam loss with no other extra beam loss by expanding the transverse painting area, it is essential to correct the beta function beating during injection. To this end, we have recently introduced six sets of pulse-type quadrupole correctors, which work as per the time dependence of the injection bump during injection [12].

In this paper, we present recent experimental results on the correction scheme to suppress the extra beam loss that occurs when applying wide-ranging transverse painting, together with detailed discussions on the beam loss and its mitigation mechanisms based on numerical simulations.

\section{TRANSVERSE INJECTION PAINTING}

Figure 2 shows a schematic view of the RCS injection section. As shown in the figure, two types of orbit-bump systems are used for beam injection $[13,14]$. One is the shift-bump system (four horizontal pulse dipole magnets; SB1-4) to produce a flattop field during injection, forming a horizontal orbit bump offset at the injection point where the charge-exchange foil is installed. The other is the paint-bump system (four horizontal and two vertical pulse dipole magnets; PBH1-4 and PBV1-2) to generate a time dependent bump orbit at the injection point for beam painting.

Figure 3 shows a schematic diagram of the injection painting process on the horizontal and the vertical phase spaces. In the horizontal painting process, $\mathrm{PBHs}$, which are installed in the ring, produce a horizontal closed orbit variation at the injection point, by which the injected beam is distributed from the middle to the outside in the circulating beam ellipse along its major axis. On the other hand, in the vertical painting process, $\mathrm{PBVs}$, which are installed in the injection beam transport line nearly 180-degrees upstream of the injection point in betatron phase advance, produce a vertical injection angle change at the injection point, by which the injected beam is similarly filled from the middle to the outside in the circulating beam ellipse along its angular axis. For this painting process, the phase-space offset of the injection beam relative to the ring closed orbit varies as per the following square-root-type functions: 


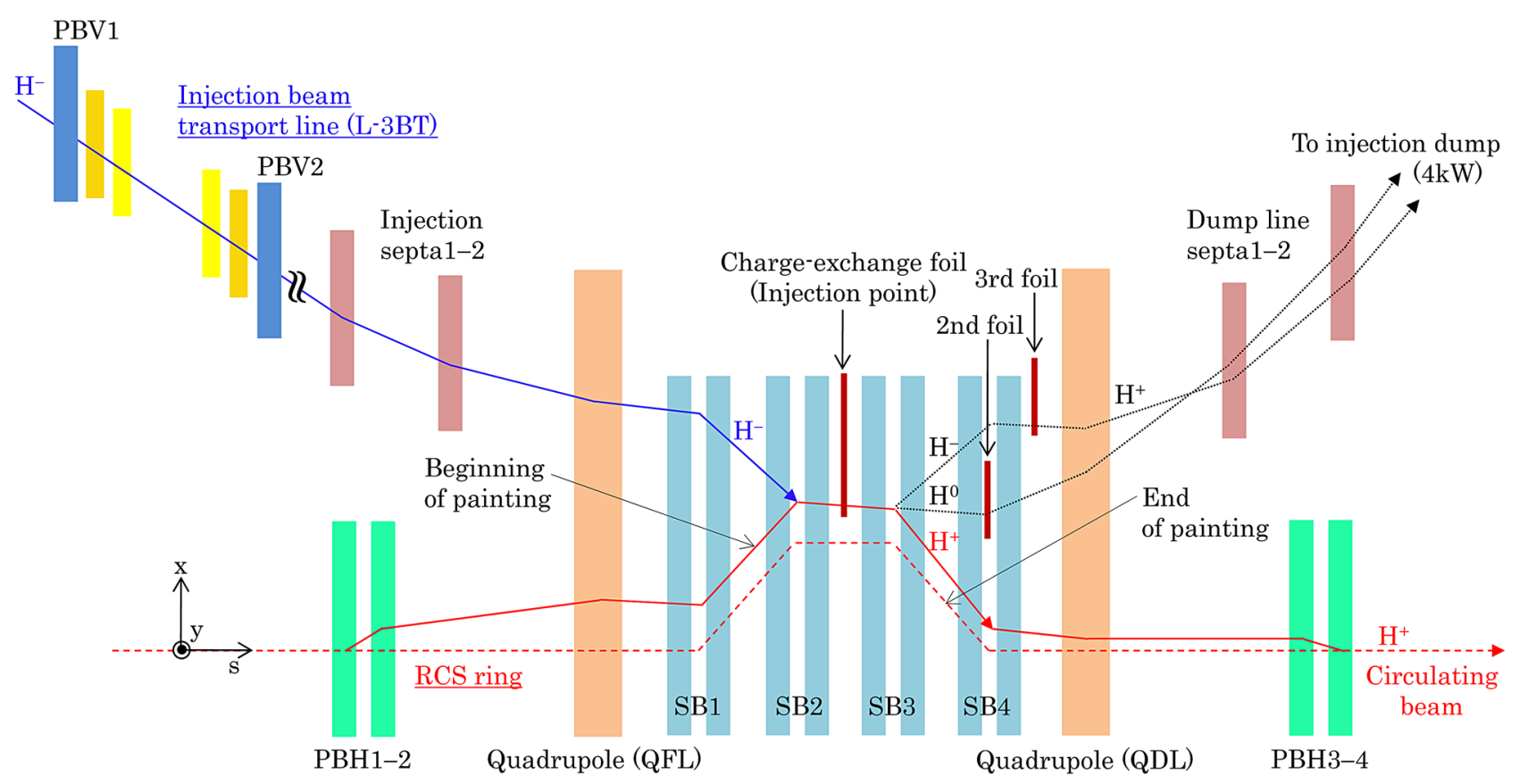

FIG. 2. Schematic view of the RCS injection section.

$$
\begin{aligned}
& x_{\text {paint }}=x_{\max } \sqrt{t / T}, \\
& x_{\text {paint }}^{\prime}=-x_{\max }^{\prime} \sqrt{t / T},
\end{aligned}
$$

for the horizontal plane, and

$$
\begin{aligned}
& y_{\text {paint }}=0, \\
& y_{\text {paint }}^{\prime}=-y_{\max }^{\prime} \sqrt{t / T},
\end{aligned}
$$

for the vertical plane. Here $\left(x_{\max }, x_{\max }^{\prime}\right)$ and $\left(y_{\max }, y_{\max }^{\prime}\right)$ are the maximum phase-space offsets on the horizontal and the vertical planes corresponding to the edge of the circulating beam ellipse with a required painting emittance of $\epsilon_{t p}, T$ is the injection duration of $0.5 \mathrm{~ms}$, and $t$ is the time step from 0 through the end of injection $T$. This type of transverse painting is known as correlated painting $[15,16]$.

In addition to space-charge mitigation, transverse painting has another vital role, i.e., mitigating the foil scattering beam loss generated in proportion to the foil hitting rate during injection. As shown in Fig. 3, the foil hitting rate decreases as the horizontal painting area becomes wider, because the circulating beam escapes more rapidly from the foil thanks to the larger horizontal closed orbit variation in the painting process. Vertical painting also acts to reduce the foil hitting rate through the wider painting area than the vertical dimension of the foil. Thus far the painting emittance was set to $100 \pi \mathrm{mm}$ mrad as the required value to minimize space-charge induced beam loss, which corresponds to $\left(x_{\max }, x_{\max }^{\prime}\right)=(26.6 \mathrm{~mm}, 3.8 \mathrm{mrad})$ and $\left(y_{\max }, y_{\max }^{\prime}\right)=$ $(0.0 \mathrm{~mm}, 2.3 \mathrm{mrad})$. The main purpose of this work is to further reduce the foil scattering part of beam loss by expanding the transverse painting area to $150 \pi \mathrm{mm} \mathrm{mrad}$, which corresponds to $\left(x_{\max }, x_{\max }^{\prime}\right)=(35.7 \mathrm{~mm}, 5.1 \mathrm{mrad})$ and $\left(y_{\max }, y_{\max }^{\prime}\right)=(0.0 \mathrm{~mm}, 3.0 \mathrm{mrad})$. As mentioned in the last section, the key in this attempt is to suppress the extra beam loss that occurs when expanding the painting area, which is caused by beta function beating due to edge focusing of the injection bump magnets.

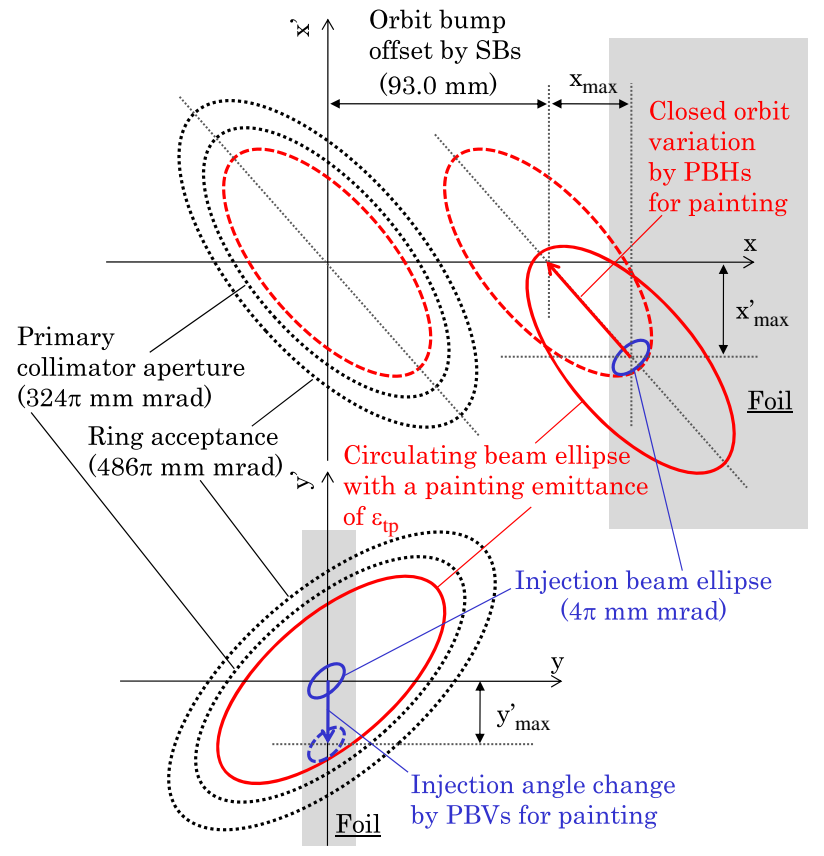

FIG. 3. Schematic diagram of transverse injection painting, where the upper plot is for the horizontal phase space, while the lower one is for the vertical one. 


\section{BETA FUNCTION BEATING CAUSED BY EDGE FOCUSING OF INJECTION BUMP MAGNETS AND ITS CORRECTION}

As mentioned in the last section, beam injection in RCS is performed with a time-dependent horizontal local orbit bump, which is formed by eight sets of pulse-type rectangular dipole magnets SB1-4 and PBH1-4. The time dependence over the $150 \pi \mathrm{mm}$ mrad transverse painting process is displayed in Fig. 4, which is formed by the excitation field forms of SBs and PBHs given in Fig. 5. As shown in Figs. 4 and 5, horizontal injection painting is realized by the local orbit bump variation from $T_{0}$ to $T_{5}$ by PBHs. Then, after the beam injection, the local orbit bump offset by SBs falls linearly from $T_{5}$ to $T_{9}$. This injection method produces edge angles at the entrance and exit of each SB and PBH as per the time dependence of the local orbit bump during the first $0.85 \mathrm{~ms}$ from $\mathrm{T}_{0}$ to $\mathrm{T}_{9}$. As is well known, each edge angle generates additional focusing force like a quadrupole magnet, but the horizontal edge focus effect is compensated by another horizontal focusing property which is intrinsic on the bending plane. Therefore, the edge focus of the injection bump magnets causes beta function beating only on the vertical plane through the remaining vertical edge focus. This is a characteristic feature of the edge focus effect, which is different from that of a quadrupole (detailed explanation of the edge focus effect is given in Ref. [12]).

Thus, the edge focus of the injection bump magnets distorts the lattice superperiodicity only on the vertical plane. Therefore, it selectively excites or enhances random

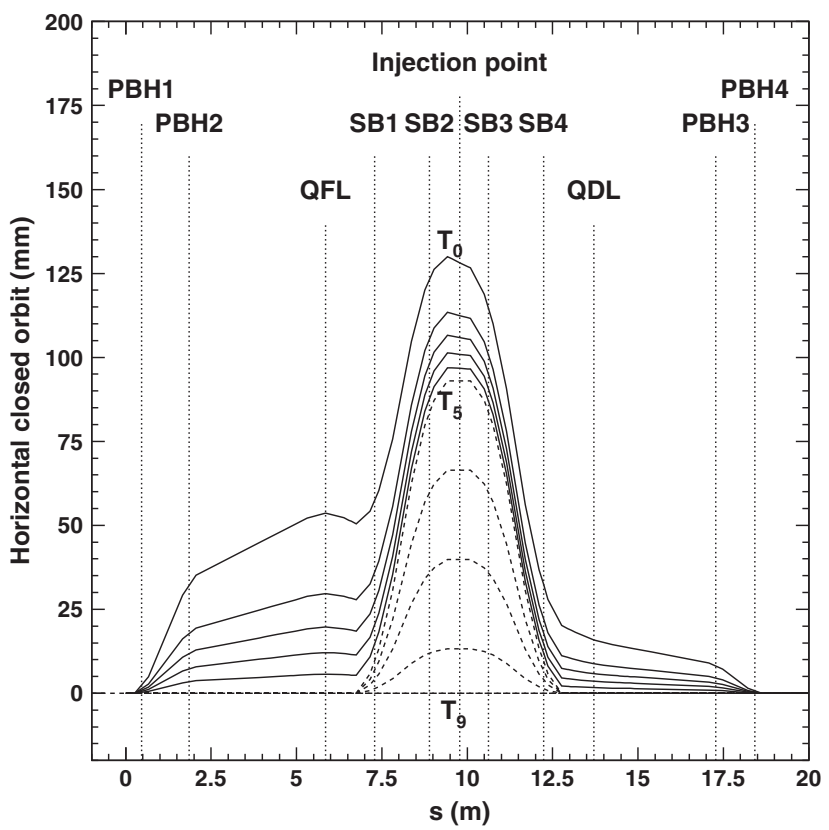

FIG. 4. Time dependence of the horizontal local orbit bump formed for $150 \pi \mathrm{mm}$ mrad transverse painting, where $\mathrm{T}_{n}(n=0-9)$ is defined in Fig. 5.

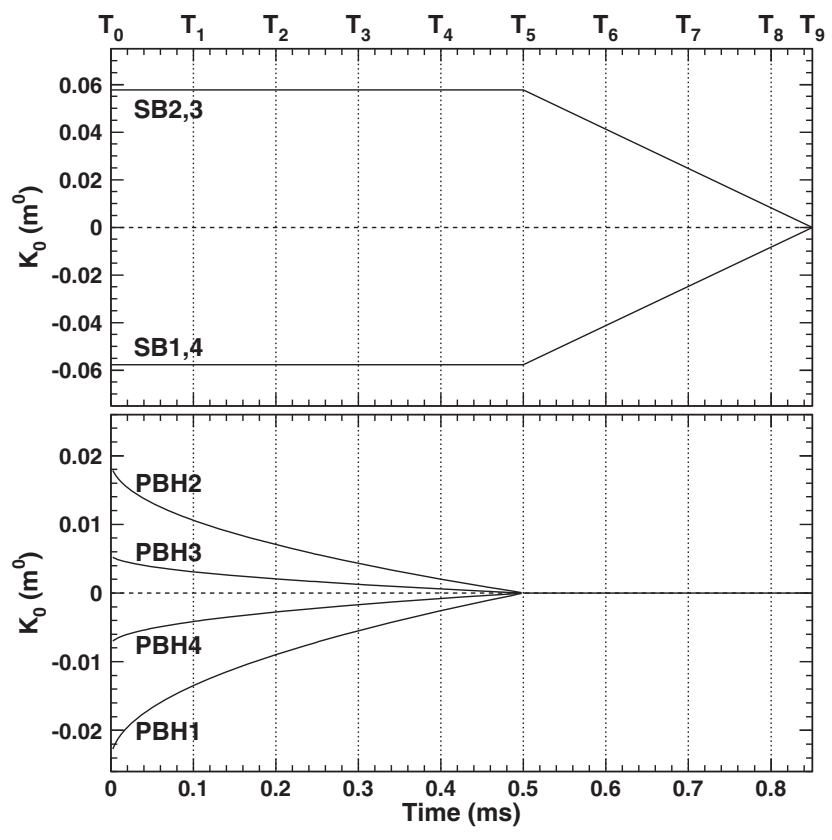

FIG. 5. Excitation field forms of SBs (top) and PBHs (bottom) required for $150 \pi \mathrm{mm}$ mrad transverse painting, where $\mathrm{k}_{0}$ is defined as $\int\left(B_{y} / B \rho\right) d s$, where $B \rho$ is the momentum rigidity.

betatron resonances related to the vertical motion and its coupling to the horizontal motion. Such additional random betatron resonances cause extra beam loss when performing wide-ranging transverse painting.

The key to solving the above issue is to repair the superperiodic condition by compensating beta function beating, by which the effects of the random resonances can be mitigated. For this purpose, we have recently introduced six sets of quadrupole correctors (QDT1-6) with independent pulse-type power supplies [12]. The installation sites of QDTs are shown by red circles in Fig. 1, which are located at both ends of each dispersionfree long straight insertion. Figure 6 shows the field forms of QDTs required for correcting the beta function beating in the case of $150 \pi \mathrm{mm}$ mrad painting. In this figure, the time

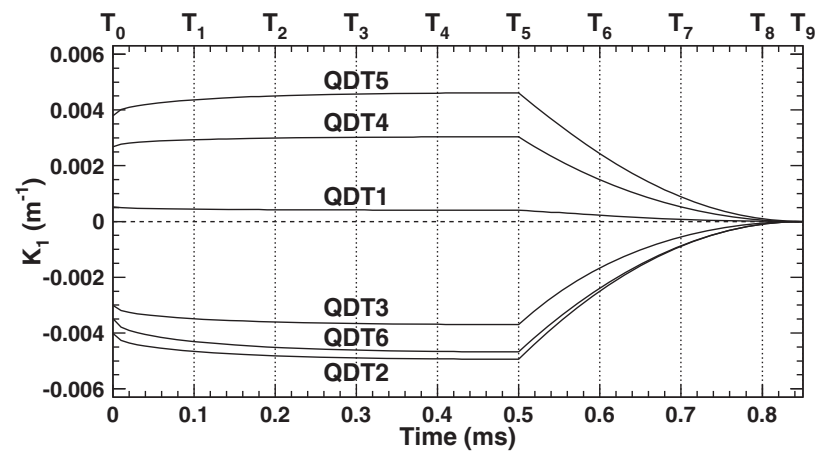

FIG. 6. Excitation field forms of QDTs required for correcting beta function beating in the case of $150 \pi \mathrm{mm}$ mrad transverse painting, where $k_{1}$ is defined as $\int\left(B_{y}^{\prime} / B \rho\right) d s$. 

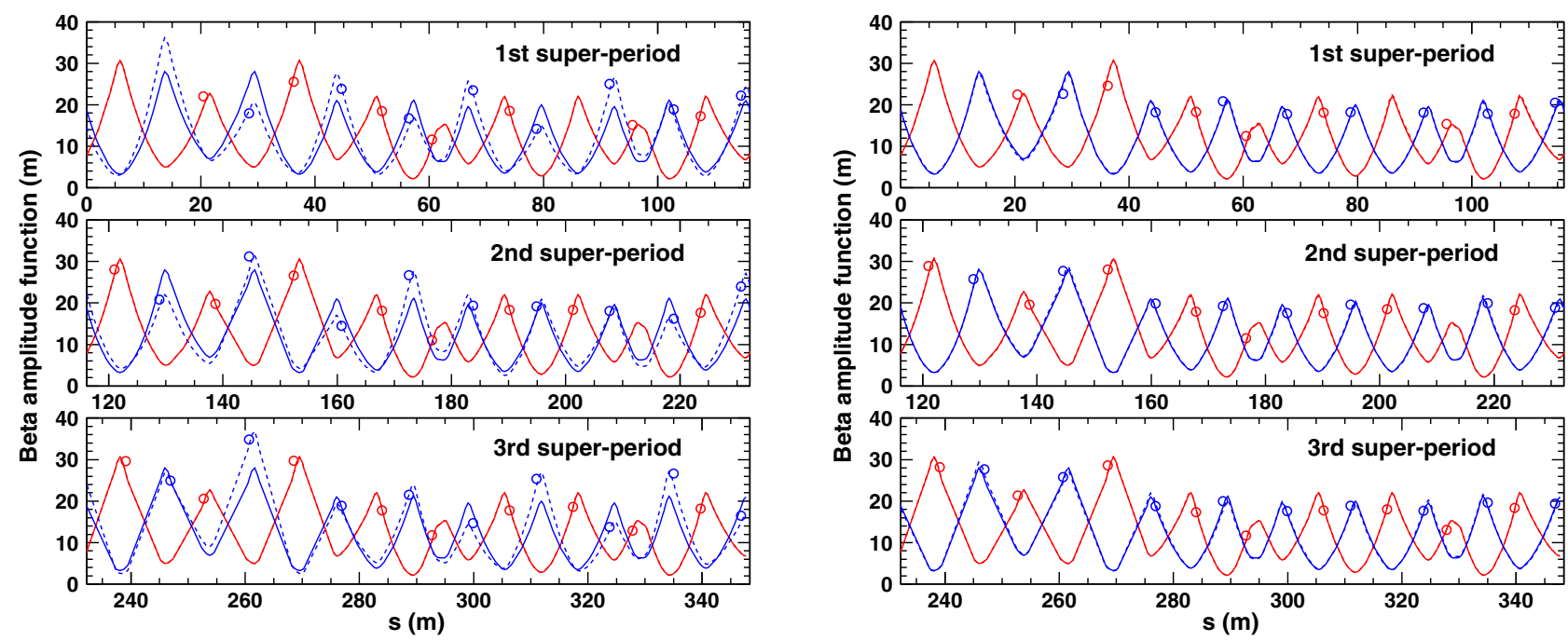

FIG. 7. Horizontal (red circles) and vertical (blue circles) beta functions measured without (left) and with (right) QDTs when the injection bump is active, where the dashed curves are the corresponding calculated results, while the solid ones are the unmodulated beta functions calculated with no injection bump.

dependences of the field forms between $T_{0}$ and $T_{5}$ reflect the bump orbit variation by $\mathrm{PBH}$ for injection painting, while those between $T_{5}$ and $T_{9}$ arise from the linear fall in the orbit bump offset by SBs.

Figure 7 shows recent experimental results concerning beta function correction by QDTs. As shown in the figure, the edge focus led to a considerable $35 \%$ beta function beating on the vertical plane, but most of it was effectively corrected by QDTs to less than 5\%, while sufficiently maintaining the superperiodic condition of the horizontal beta function.

\section{EXPERIMENTAL RESULTS AND DISCUSSIONS WITH NUMERICAL SIMULATIONS}

In June 2015, we conducted a beam experiment to confirm the effectiveness of the above correction scheme, and compared the experimental results with the corresponding numerical simulation results to clarify the detailed mechanisms of beam loss and its mitigation.

\section{A. Experimental condition}

The beam test was performed with single-shot beam-ondemand operation using a $0.5 \mathrm{~ms}-$ long injection pulse with a peak current of $37.6 \mathrm{~mA}$ and a chopper beam-on duty factor of $60 \%$. The number of particles per pulse was then $7.05 \times 10^{13}$, which corresponds to an output beam power of $\sim 850 \mathrm{~kW}$ at the design repetition rate of $25 \mathrm{~Hz}$. In this beam test, the operational bare tune $\left(\nu_{x}, \nu_{y}\right)$ was set to $(6.45$, 6.38). This operating point and its vicinity are promising candidates for the 1-MW design beam operation, which allow incoherent tune shifts to avoid serious systematic resonances.

\section{B. Numerical simulation setup}

The corresponding numerical simulation was performed with a particle-tracking code called "SimPSONS" [17]. This code enables us to simulate the three-dimensional motion of beam particles, including the space-charge effect and the realistic injection painting process, and it has well reproduced the experimental results obtained so far in RCS, such as beam loss, beam profile, bunching factor, and their time and intensity dependences $[4,18]$.

In this code, all lattice elements are represented as thin lens elements. The conversion from thick to thin lens descriptions is performed with another code called "TEAPOT" [19]. At this stage, lattice imperfections such as field and alignment errors are reflected in the thin lens representation. In the present simulation, all lattice imperfections identified thus far were included in addition to the edge focus of the injection bump magnets; multipole field components of all ring magnets, magnetic field errors, misalignments, static leakage fields from extraction beamline magnets, dipole field ripples of main bending magnets, and so on. One characteristic feature of this code is that the independent variable is time, not longitudinal position, as is the case generally. Therefore, time-dependent lattice imperfections, such as the present issue of the edge focusing effect during injection, can be easily included in the simulation.

This code computes the space-charge potential using the particle-in-cell algorithm with cylindrical meshes in the $(r, \theta, z)$ coordinates, applying the space-charge force to each macroparticle as a three-dimensional impulse kick. The space-charge potential is recalculated at a certain time step out of the macroparticles whose distribution evolves in a self-consistent manner as per the progression of time. The time step applied in the present simulation was 2 ns during 
injection and $10 \mathrm{~ns}$ for the others, which corresponds to 850 and 170 kicks per turn. The number of macroparticles in this work was set to $5 \times 10^{5}$ using a transverse grid of $64(r) \times 64(\theta)$ for the conducting boundary of $r=0.145 \mathrm{~m}$, and a longitudinal grid of $50(z)$. The careful investigation for the convergence of macroparticle motions confirmed that the use of these numerical parameters is tolerable to obtain meaningful simulation results in this paper.

\section{Results and discussions}

Figure 8 shows the scintillation-type beam loss monitor (BLM) signals over the first $1.4 \mathrm{~ms}$ measured in the collimator section under various operational conditions. The top plot shows the case of the original painting emittance of $100 \pi \mathrm{mm}$ mrad, while the middle plot corresponds to the result of employing the wider transverse painting area of $150 \pi \mathrm{mm}$ mrad. As is obvious from their comparison, significant beam loss occurs when expanding the painting area from $100 \pi$ to $150 \pi \mathrm{mm} \mathrm{mrad}$, with the beam loss rate estimated to be $0.4 \%$. But the extra beam loss was nearly minimized as expected by introducing QDTs, as shown in the bottom plot. These empirical results were well reproduced by the corresponding numerical simulations, as shown in Fig. 9, while the calculated extra beam loss lowers by several $10 \%$.

Figures 10 and 11 show the tune footprints and the twodimensional transverse phase space coordinates at the end of injection calculated for the painting emittances of $100 \pi$ and $150 \pi \mathrm{mm}$ mrad, where three kinds of transverse beam distributions are given: (a) without edge focus, (b) with edge focus, and (c) with the addition of QDTs to (b). In the case of the painting emittance of $100 \pi \mathrm{mm}$ mrad, there is no significant difference among (a), (b), and (c), but in the

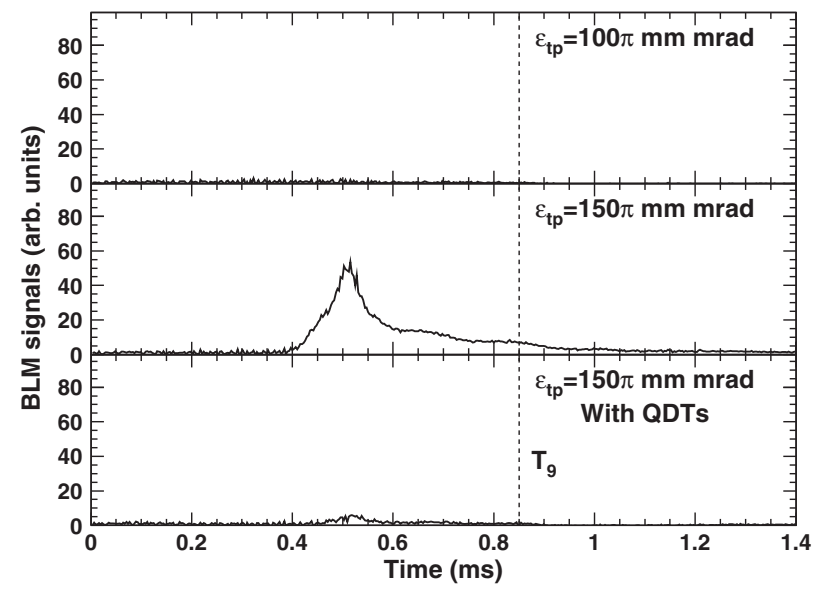

FIG. 8. Scintillation-type BLM signals over the first $1.4 \mathrm{~ms}$ measured in the collimator section with a beam intensity of $850 \mathrm{~kW}$; (top) $100 \pi \mathrm{mm}$ mrad transverse painting, (middle) $150 \pi \mathrm{mm} \mathrm{mrad}$ transverse painting, and (bottom) $150 \pi \mathrm{mmmrad}$ transverse painting with QDTs.

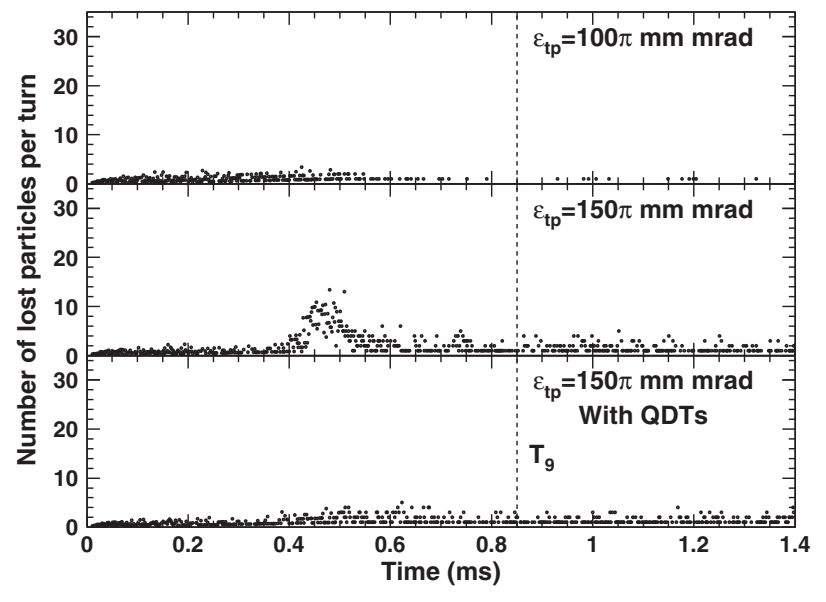

FIG. 9. Numerical simulation results corresponding to the experimental data in Fig. 8.

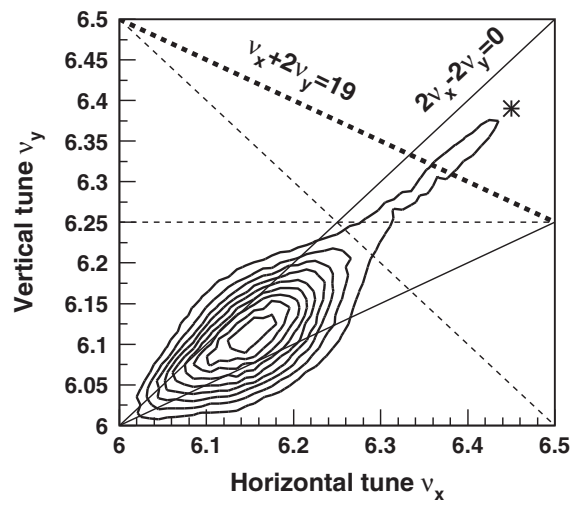

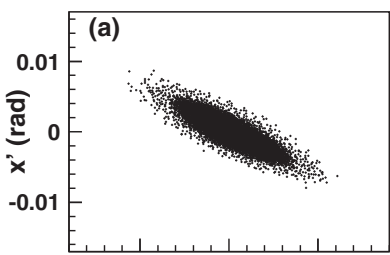
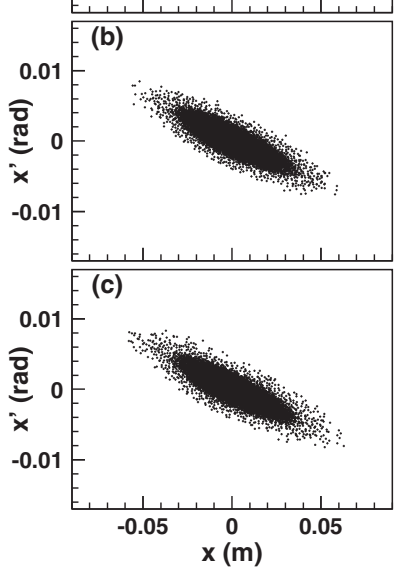

(a)

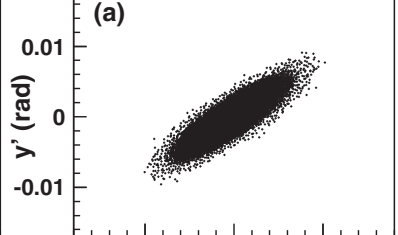

(b)

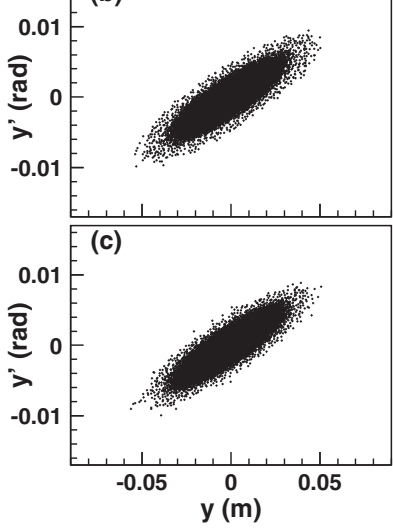

FIG. 10. Tune footprint (top) and two-dimensional transverse phase space coordinates (bottom) at the end of injection, calculated with a beam intensity of $850 \mathrm{~kW}$ in the case of the painting emittance of $100 \pi \mathrm{mm} \mathrm{mrad}$, where three kinds of transverse beam distributions are given: (a) without edge focus, (b) with edge focus, and (c) with the addition of QDTs to (b). 

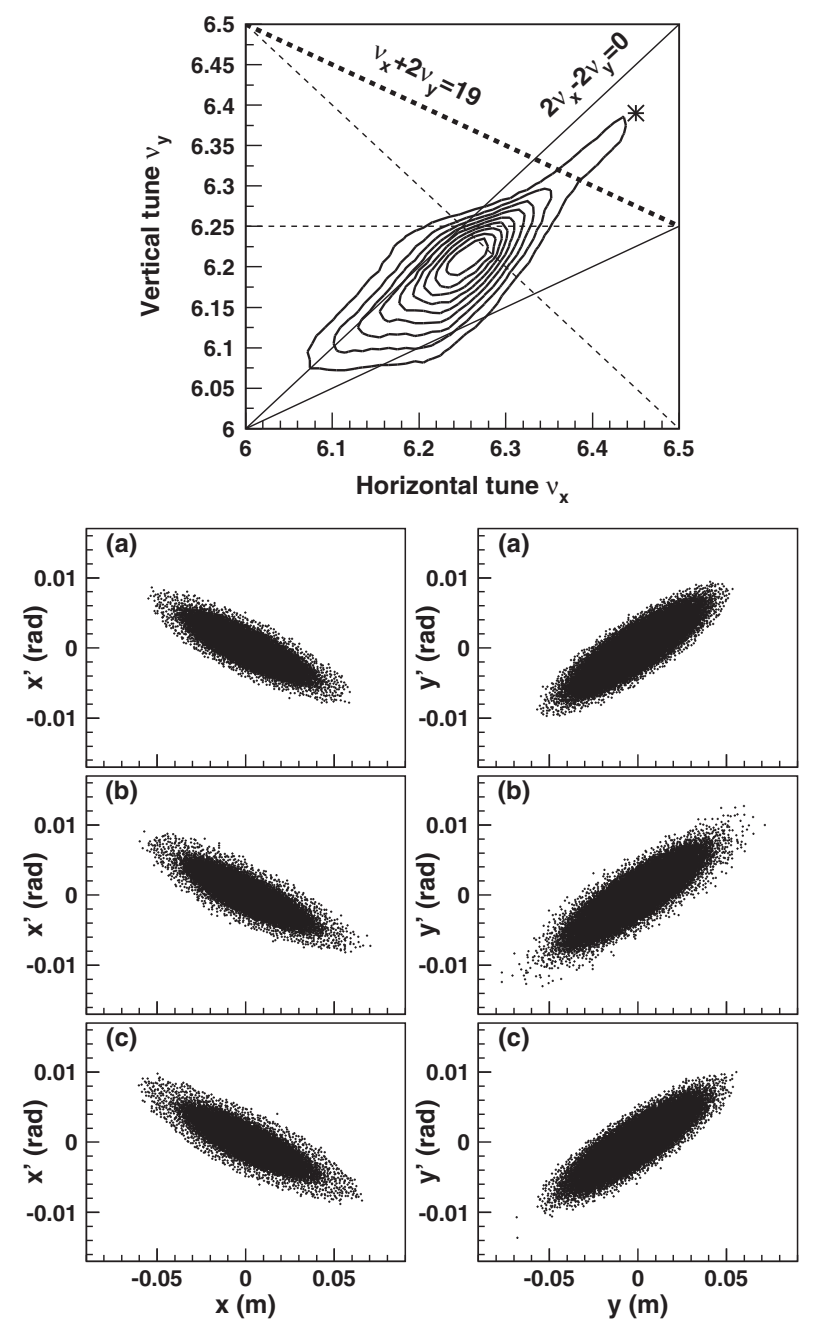

FIG. 11. Similar results to those in Fig. 10, calculated with a beam intensity of $850 \mathrm{~kW}$ in the case of the painting emittance of $150 \pi \mathrm{mm}$ mrad: (a) without edge focus, (b) with edge focus, and (c) with the addition of QDTs to (b).

case of the painting emittance of $150 \pi \mathrm{mm}$ mrad, one can find that beam halo formation is enhanced from (a) to (b) by the edge focus, especially on the vertical plane. This beam halo formation causes the extra beam loss observed in the middle plot in Fig. 8. But, the beam halo is well mitigated from (b) to (c) by QDTs, which corresponds to the beam loss reduction observed in the bottom plot in Fig. 8.

The beam halo formation arises from random betatron resonances, which are additionally excited by the edge focus during injection. The edge focus of the injection bump magnets distorts the lattice superperiodicity only on the vertical plane. Therefore, it selectively excites random resonances related to the vertical motion and its coupling to the horizontal motion. The dashed lines in the tune diagrams in Figs. 10 and 11 correspond to such random resonances, where the resonances are plotted up to the fourth order. The present operating point is set to (6.45, 6.38), as shown by an asterisk in the tune diagram, which is far from the random resonances, but some beam particles cross them due to the space-charge tune shift.

To identify the most critical random resonance for beam halo formation, the single-particle behavior of each macroparticle was investigated. Figure 12 shows a typical sample of the turn-by-turn incoherent motion of one macroparticle that forms the beam halo observed in Fig. 11(b), where the top plot shows the turn-by-turn incoherent position oscillations, while the bottom plot is their two-dimensional transverse phase-space coordinates. Turn-by-turn betatron actions $J_{x}$ and $J_{y}$ obtained from the incoherent oscillations are given in Fig. 13. As shown in the figure, the betatron actions gradually increase on both the horizontal and the vertical planes while oscillating. This single-particle behavior can be interpreted as the combined effect of two resonances $\nu_{x}+2 \nu_{y}=19$ and $2 \nu_{x}-2 \nu_{y}=0$. Figure 14 shows a two-dimensional plot of the turn-by-turn incoherent tunes calculated from the incoherent oscillations given in Fig. 12. In this figure, one can see that the incoherent tunes are located on the $\nu_{x}+2 \nu_{y}=19$ resonance as well as near the $2 \nu_{x}-2 \nu_{y}=0$ resonance. The $\nu_{x}+2 \nu_{y}=19$ resonance is a third-order random resonance arising from the chromatic correction sextupole field and the intrinsic sextupole field component in the main bending magnets, and it is additionally excited by the edge focus during injection. This nonlinear sum resonance induces emittance growth on both the horizontal and the vertical planes, while $2 J_{x}-J_{y}$ is conserved [20]. Specifically, this resonance leads to 2 times larger emittance growth on the vertical
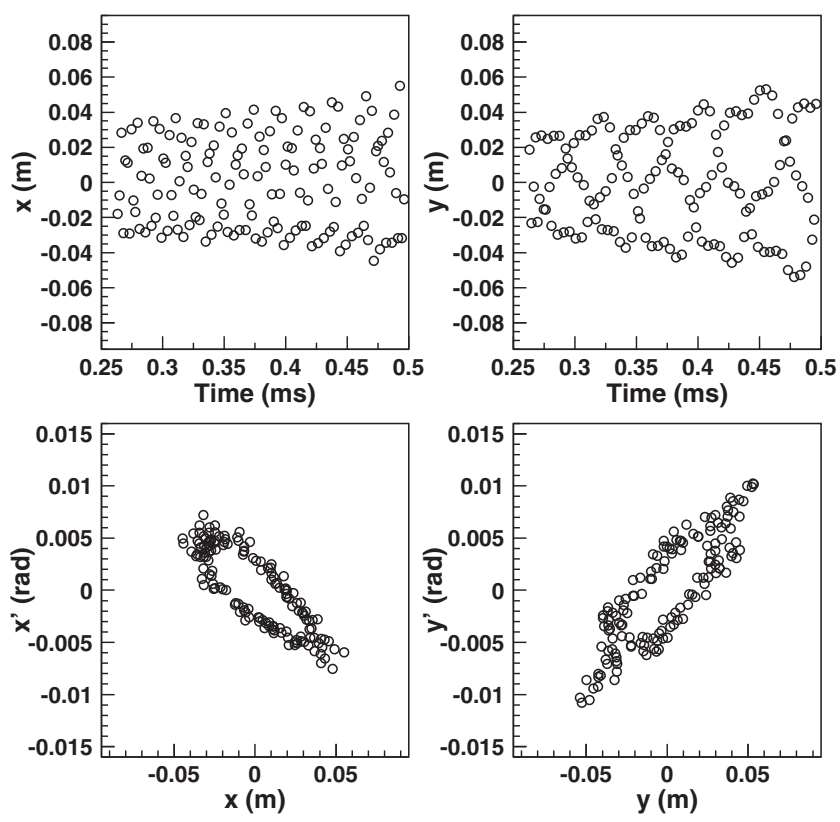

FIG. 12. Typical sample of the incoherent motion of one macroparticle that forms beam halo, where the top plot shows the turn-by-turn incoherent position oscillations, while the bottom plot is their two-dimensional transverse phase-space coordinates. 


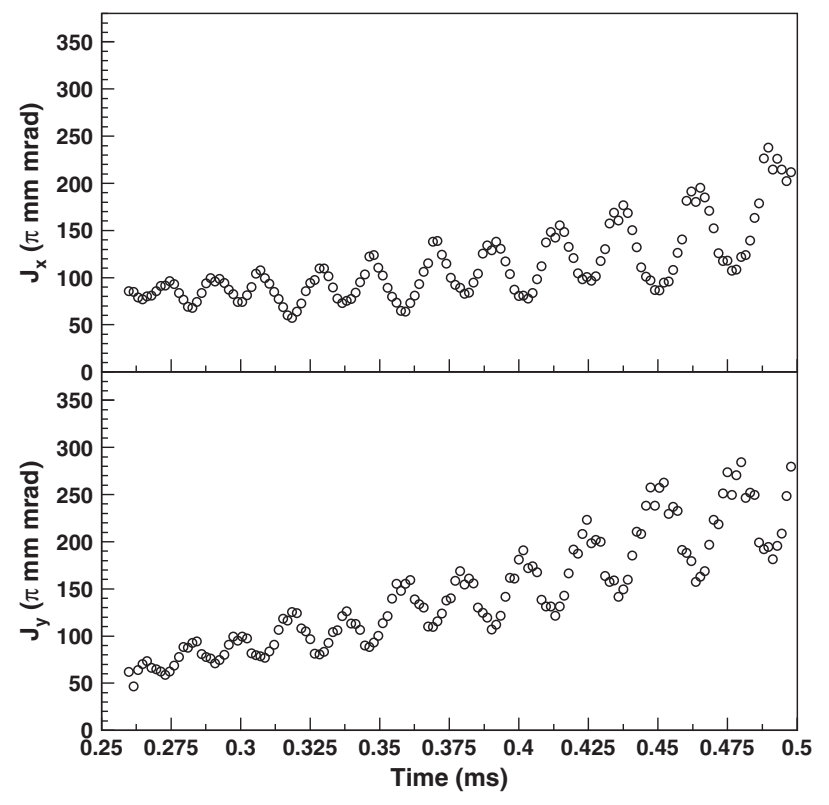

FIG. 13. Turn-by-turn betatron actions, calculated from the particle oscillations given in Fig. 12.

plane than that on the horizontal plane. On the other hand, the $2 \nu_{x}-2 \nu_{y}=0$ resonance is a fourth-order systematic resonance, which mainly arises from the fourth-order component in the space-charge force and the second-order effect of the sextupole field. Therefore, it affects the beam regardless of the edge focus. This nonlinear difference resonance induces emittance exchange between the horizontal and the vertical planes, while $J_{x}+J_{y}$ is conserved $[21,22]$. Figure 15 shows a two-dimensional plot of $\left(J_{x}, J_{y}\right)$ given in Fig. 13. In this figure, one can confirm the

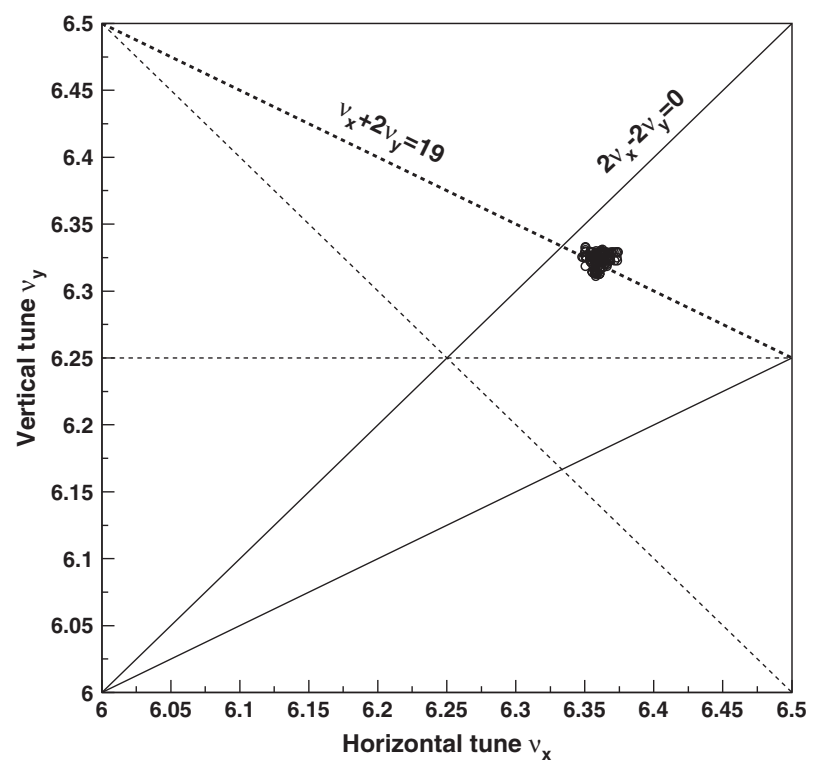

FIG. 14. Two-dimensional plot of the turn-by-turn incoherent tunes, calculated from the particle oscillations given in Fig. 12.

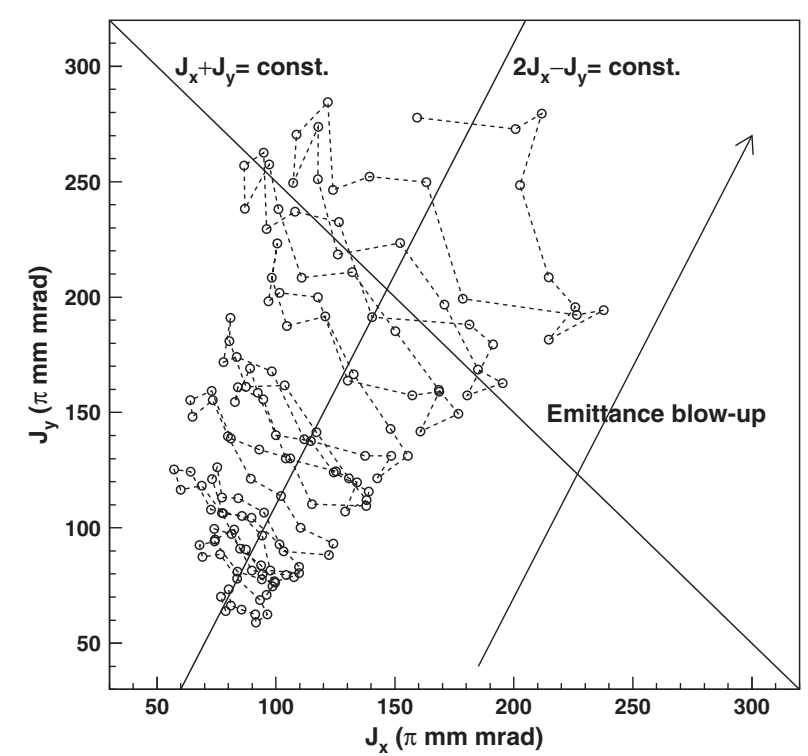

FIG. 15. Two-dimensional plot of the turn-by-turn betatron actions given in Fig. 13.

characteristic emittance blowup that implies the combined effect of the two resonances; the horizontal and the vertical actions of the macroparticle gradually grow up along the line of $2 J_{x}-J_{y}=$ const, while oscillating in a direction parallel to the line of $J_{x}+J_{y}=$ const. This analysis confirmed that most of the beam halo particles observed in Fig. 11(b) are generated through such a single-particle behavior caused by the two resonances. In addition, it revealed that the contribution of the $\nu_{x}+2 \nu_{y}=19$ resonance is more critical for the observed extra beam loss, because the resonance leads to more severe emittance growth on the vertical plane. Actually, as shown in Fig. 8, the extra beam loss was well minimized by mitigating the effect of the $\nu_{x}+2 \nu_{y}=19$ resonance with QDTs, where the effect of the $2 \nu_{x}-2 \nu_{y}=0$ resonance hardly changes.

Another question left is why beam halo formation is more enhanced in the case of $150 \pi \mathrm{mm}$ mrad transverse painting. This is due to the different situation of resonance crossing to $\nu_{x}+2 \nu_{y}=19$. As shown by the tune footprint in Fig. 10, the number of beam particles on the resonance is less for narrower transverse painting of $100 \pi \mathrm{mm} \mathrm{mrad}$ thanks to the larger space-charge tune shift. On the other hand, in the case of wider transverse painting of $150 \pi \mathrm{mm} \mathrm{mrad}$, that number increases due to its resultant tune shift mitigation, as shown by the tune footprint in Fig. 11. This is the main reason why the effect of the resonance is more critical in the case of $150 \pi \mathrm{mm} \mathrm{mrad}$ transverse painting. To deepen our understanding of this phenomenon, we performed another measurement with the painting emittance of $150 \pi \mathrm{mm} \mathrm{mrad}$, where the beam intensity was slightly reduced from 850 to $740 \mathrm{~kW}$. The BLM signals in this case are shown in Fig. 16. In the 


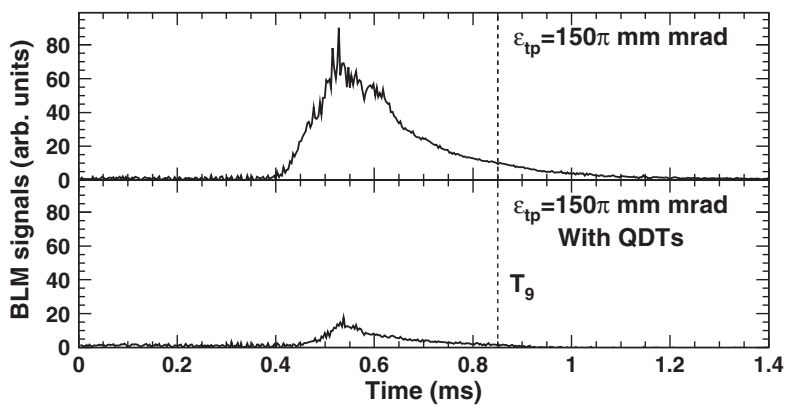

FIG. 16. Scintillation-type BLM signals over the first $1.4 \mathrm{~ms}$ measured in the collimator section with a beam intensity of $740 \mathrm{~kW}$; (top) $150 \pi \mathrm{mm}$ mrad transverse painting, and (bottom) $150 \pi \mathrm{mm}$ mrad transverse painting with QDTs.

comparison of Figs. 8 (middle) and 16 (top), one can see that the beam loss before the addition of QDTs is enhanced through the decrease of the beam intensity; the beam loss rate increased by a factor of 1.7. This empirical situation was also well reproduced by the numerical simulations, as shown in Fig. 17. Figure 18 shows the similar results to those given in Fig. 11, calculated with the reduced beam intensity of $740 \mathrm{~kW}$. As is obvious from the comparison of Figs. 11 and 18, the beam halo formation from (a) to (b) is enhanced by reducing the beam intensity, which corresponds to the beam loss increase observed in Fig. 16. This phenomenon can also be interpreted by the similar reason that the decrease of the beam intensity increases the number of particles trapped in the $\nu_{x}+2 \nu_{y}=19$ resonance through its resultant tune shift mitigation, as shown by the tune footprint in Fig. 18. The enhanced extra beam loss was also significantly mitigated with the addition of QDTs, as shown in the bottom plot in Fig. 16. In this case, small beam loss still remains even with QDTs due to the present correction accuracy, but it will be minimized to the same level as the $850-\mathrm{kW}$ case by slightly moving the bare tune so that the number of particles on the resonance decreases, e.g., from $(6.45,6.38)$ to $(6.45,6.36)$.

Finally, we investigated the effect of the sextupole fields on the extra beam loss. Figure 19 shows the BLM signals measured with the painting emittance of $150 \pi \mathrm{mm} \mathrm{mrad}$

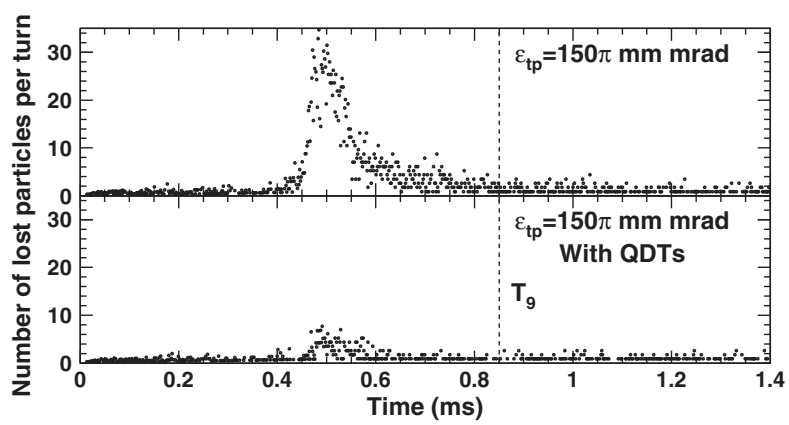

FIG. 17. Numerical simulation results corresponding to the experimental data in Fig. 16.
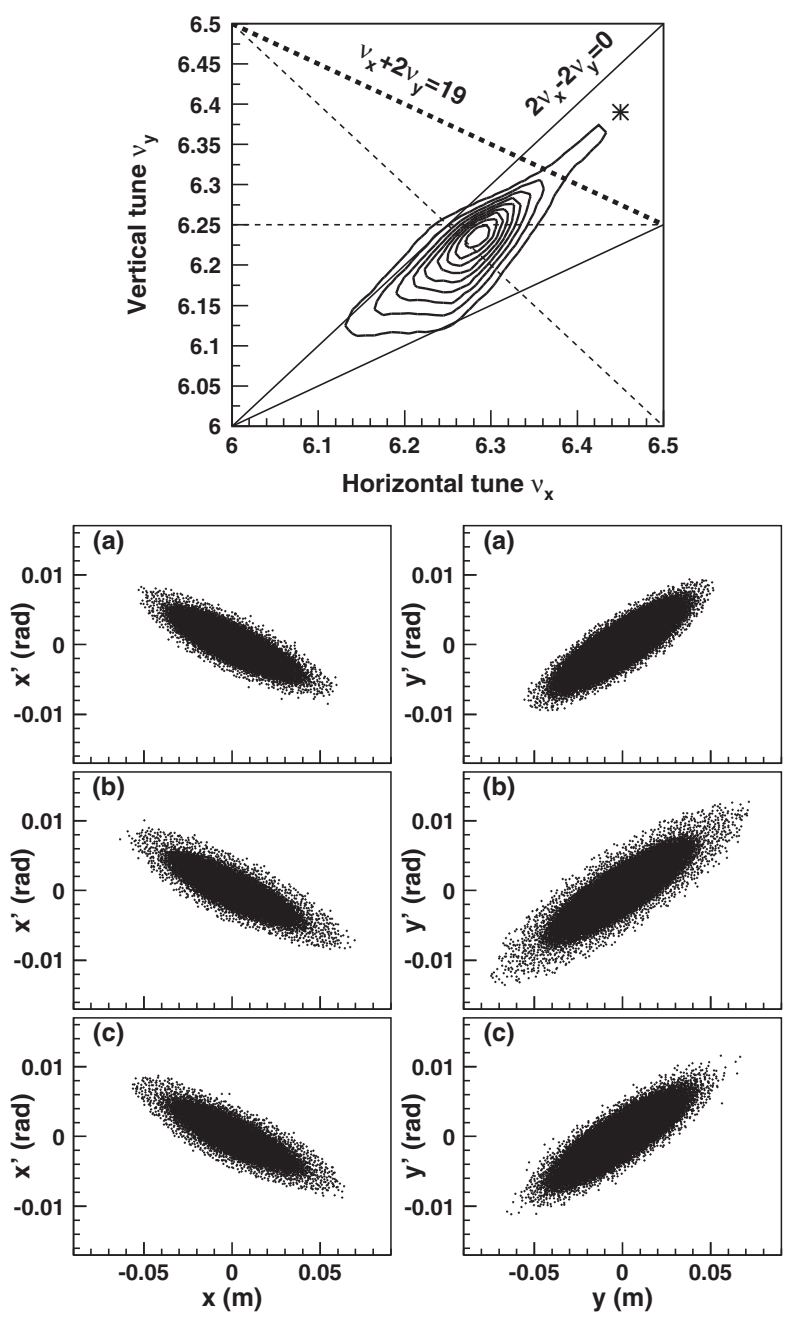

FIG. 18. Similar results to those in Fig. 11, calculated with a beam intensity of $740 \mathrm{~kW}$ in the case of the painting emittance of $150 \pi$ mm mrad: (a) without edge focus, (b) with edge focus, and (c) with the addition of QDTs to (b).

for a beam intensity of $740 \mathrm{~kW}$, where the chromaticity correction sextupole fields were reduced by half. As is obvious from the comparison to Fig. 16, the extra beam loss without QDTs decreased as expected as per the reduction of the sextupole field strengths. The experimental data additionally support our conclusions that the third-order random resonance $\nu_{x}+2 \nu_{y}=19$ is the main cause of the extra beam loss, and that the mitigation of the $\nu_{x}+2 \nu_{y}=19$ resonance achieved with QDTs leads to the significant beam loss mitigation. Whereas a saving of sextupole fields favors the mitigation of the $\nu_{x}+2 \nu_{y}=19$ resonance, at least half the chromaticity correction will be essential to minimize some other beam losses than that, for a variety of beam conditions of up to $1 \mathrm{MW}$. QDTs play a significant role also in improving the flexibility of the use of sextupole fields.

Through these series of beam experiments and simulations, we confirmed that the flexibility of transverse 


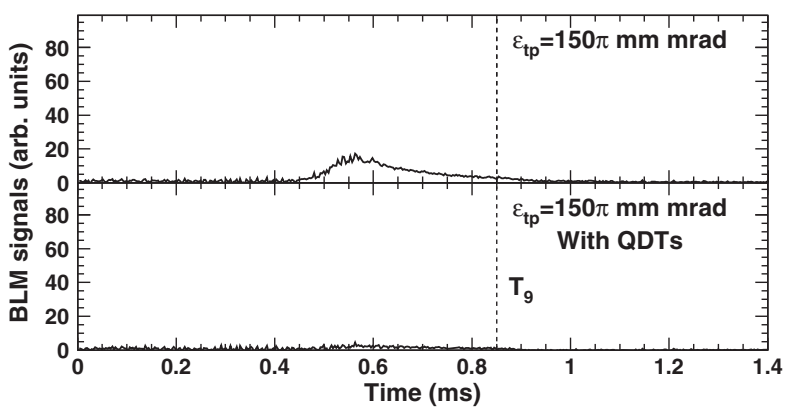

FIG. 19. Scintillation-type BLM signals over the first $1.4 \mathrm{~ms}$ measured in the collimator section with a beam intensity of $740 \mathrm{~kW}$, where the chromaticity correction sextupole fields were reduced by half: (top) $150 \pi \mathrm{mm} \mathrm{mrad}$ transverse painting, and (bottom) $150 \pi \mathrm{mm}$ mrad transverse painting with QDTs.

painting area is improved as expected by QDTs, and also clarified the detailed mechanisms on beam halo and beam loss caused by resonance crossing. The correction accuracy for beta function beating can be further improved by increasing the number of quadrupole correctors, while the present performance is estimated to be enough to realize sufficient beam loss mitigation.

\section{SUMMARY}

Beta function beating during injection, caused by edge focusing of pulsed injection bump magnets, additionally excites random betatron resonances through a distortion of the lattice superperiodicity, leading to significant extra beam loss when applying wide-ranging transverse injection painting. To solve this issue, we newly installed six sets of pulse-type quadrupole correctors.

The beta function beating was well compensated with the quadrupole correctors, by which the transverse painting area was successfully expanded from $100 \pi$ to $150 \pi \mathrm{mm}$ mrad with no significant extra beam loss for a beam intensity of $850 \mathrm{~kW}$. This achievement leads to sufficient mitigation of the foil scattering beam loss as well as minimizing the space-charge induced beam loss; the average number of foil hits per particle during injection was reduced from 42 to 26 by the expansion of the transverse painting area, and then further to 12 in combination with additional adjustments for the foil size and its positioning. By such recent efforts, the 1-MW beam operation is now estimated to be established within the permissible beam loss level. In addition, the characteristic mechanisms of beam halo and beam loss caused by resonance crossing were revealed through a series of beam experiments and simulations in this work.

Beta function beating during injection will be a common issue in high-power proton rings like RCS, because transverse painting is an essential technique in such facilities. The present correction scheme is straightforward, and can be applied to any ring accelerators.

\section{ACKNOWLEDGMENTS}

The authors acknowledge Dr. M. Kinsho, Dr. K. Hasegawa, Professor T. Koseki, and all the RCS group members for their continuous support and encouragement for the present work. We are also grateful to Dr. S. Meigo for his indispensable support during the beam test and to Dr. S. Machida for his valuable advice and support in improving the numerical simulation code.

[1] JAERI Report No. JAERI-Tech 2003-044, KEK Report No. 2002-13.

[2] I. Sugai, Y. Takeda, M. Oyaizu, H. Kawakami, Y. Irie, A. Takagi, H. Hattori, and K. Kawasaki, Double-layer effects on the lifetime of newly developed HBC-foils for RCS of J-PARC, Nucl. Instrum. Methods Phys. Res., Sect. A 613, 457 (2010).

[3] H. Hotchi et al., Beam commissioning of the 3-GeV rapid cycling synchrotron of the Japan Proton Accelerator Research Complex, Phys. Rev. ST Accel. Beams 12, 040402 (2009).

[4] H. Hotchi, H. Harada, N. Hayashi, M. Kinsho, P. K. Saha, Y. Shobuda, F. Tamura, K. Yamamoto, M. Yamamoto, M. Yoshimoto, and Y. Irie, Beam loss reduction by injection painting in the 3-GeV rapid cycling synchrotron of the Japan Proton Accelerator Research Complex, Phys. Rev. ST Accel. Beams 15, 040402 (2012).

[5] H. Hotchi, H. Harada, N. Hayashi, M. Kinsho, P. K. Saha, Y. Shobuda, F. Tamura, K. Yamamoto, M. Yamamoto, M. Yoshimoto, S. Kato, Y. Irie, T. Koseki, Y. Sato, K. Satou, and M. Shirakata, Beam commissioning and operation of the Japan Proton Accelerator Research Complex $3-\mathrm{GeV}$ rapid cycling synchrotron, Prog. Theor. Exp. Phys. 2012, 02B003 (2012).

[6] H. Hotchi, Commissioning the $400 \mathrm{MeV}$ linac at J-PARC and high intensity operation of the J-PARC RCS, in Proceedings of the 2014 International Particle Accelerator Conference (IPAC2014), Dresden, Germany, 2014 (JACOW, Dresden, Germany, 2014), pp. 899-903.

[7] H. Hotchi, Lessons from 1-MW proton RCS beam tuning, in Proceedings of the 54th ICFA Advanced Beam Dynamics Workshop on High-Intensity and High-Brightness Hadron Beams (HB2014), East Lansing, MI, USA, 2014 (JACOW, East Lansing, MI, USA, 2014), pp. 6-11.

[8] H. Hotchi, Recent progress of J-PARC RCS beam commissioning - toward realizing the 1-MW output beam power, in Proceedings of the 2015 International Particle Accelerator Conference (IPAC2015), Richmond, VA, USA, 2015, TUBB3 (JACOW, Richmond, VA, USA, 2015), pp. 1346-1348.

[9] E. Yamakawa, M. Yoshimoto, and M. Kinsho, Measurements and PHITS Monte Carlo estimations of residual activities induced by the $181 \mathrm{MeV}$ proton beam in the injection area at J-PARC RCS Ring, JPS Conf. Proc. 8, 012017 (2015)

[10] F. Tamura, M. Yamamoto, M. Yoshii, C. Ohmori, M. Nomura, A. Schnase, M. Toda, H. Suzuki, T. Shimada, K. Hara, and K. Hasegawa, Longitudinal painting with large 
amplitude second harmonic rf voltages in the rapid cycling synchrotron of the Japan Proton Accelerator Research Complex, Phys. Rev. ST Accel. Beams 12, 041001 (2009).

[11] M. Yamamoto, M. Nomura, A. Schnase, T. Shimada, H. Suzuki, F. Tamura, E. Ezura, K. Hara, K. Hasegawa, C. Ohmori, K. Takata, A. Takagi, M. Toda, and M. Yoshii, Simulation of longitudinal beam manipulation during multi-turn injection in J-PARC RCS, Nucl. Instrum. Methods Phys. Res., Sect. A 621, 15 (2010).

[12] H. Hotchi, N. Tani, and Y. Watanabe, Numerical study for beam loss occurring for wide-ranging transverse injection painting and its mitigation scenario in the J-PARC 3-GeV RCS, Nucl. Instrum. Methods Phys. Res., Sect. A 778, 102 (2015).

[13] T. Takayanagi, J. Kamiya, M. Watanabe, Y. Yamazaki, Y. Irie, J. Kishiro, I, Sakai, and T. Kawakubi, Design of the injection bump system of the $3-\mathrm{GeV}$ RCS in J-PARC, IEEE Trans. Appl. Supercond. 16, 1358 (2006).

[14] T. Takayanagi, N. Hayashi, M. Kinsho, T. Ueno, T. Togashi, K. Horino, and Y. Irie, Design and preliminary performance of the new injection shift bump power supply at the J-PARC 3-GeV RCS, IEEE Trans. Appl. Supercond. 24, 0503504 (2014).
[15] Y. Kamiya, Elementary analysis of phase space painting, in Proceedings of the 1989 Particle Accelerator Conference, Chicago, IL (IEEE, New York, 1989), pp. 660-662.

[16] J. Beebe-Wang, Y. Y. Lee, D. Raparia, J. Wei, C. R. Prior, and S. Machida, Beam properties in the SNS accumulator ring due to transverse phase space painting, in Proceedings of the European Particle Accelerator Conference, Vienna, 2000 (EPS, Geneva, 2000), pp. 1465-1467.

[17] S. Machida and M. Ikegami, Simulation of space charge effects in a synchrotron, AIP Conf. Proc. 448, 73 (1998).

[18] H. Hotchi, Space-charge induced beam loss and its mitigation in the J-PARC RCS, Space-charge 2015 workshop, Oxford, UK, 2015, https://www.cockcroft.ac.uk/ events/SpaceCharge15/.

[19] L. Schachinger and R. Talman, Teapot: A thin-element accelerator program for optics and tracking, Part. Accel. 22, 35 (1987).

[20] Y. Wang et al., Experimental results of the betatron sum resonance, in Proceedings of the 15th Particle Accelerator Conference, PAC-1993, Washington, DC, 1993 (IEEE, New York, 1993), pp. 227-229.

[21] B. W. Montague, CERN-Report No. 68-38, 1968.

[22] A. Ando, T. Suzuki, E. Takasaki, and K. Takayama, Coupling resonance $2 \nu_{h}-2 \nu_{v}=0$ in the KEK proton synchrotron, IEEE Trans. Nucl. Sci. 26, 3541 (1979). 